\title{
THE SERIAL POSITION EFFEGT IN VISUAL PROGESSING OF DIGITS PRESENTED IN RAPID SUCCESSION
}

\author{
KEN GORYO 1
}

Chiba University

\begin{abstract}
5 digits were sequentially presented to the same portion of the retina with various rates $(50-4 \mathrm{cps})$ for 5 to 10 subjects, and the interactions among the visual responses to the digits were investigated. Subject's task was to report the whole digits he had seen (Exp. I) or to answer whether a particular digit had been contained in a stimulus series or not (Exp. II). Percentage of correct responses in both experiments varied as a function of serial position in the stimulus series. The serial position curves were almost always in U-shape and the bottom of the curves shifted from anterior to posterior serial position as the rate of presentation decreased. It was concluded that there are at least two kinds of interactions (forward and backward maskings) among responses in the visual mechanism for processing the information sequentially given with high speed.
\end{abstract}

\section{INTRODUCTION}

The present study started from a very easily observable phenomenon. Suppose a digital frequency counter having four indicator tubes. When the counter is counting $1000 \mathrm{cps}$ pulses, for example, the right end indicator tube displays a digit changing one by one from zero to nine at the rate of 1000 times per second and in the second, third and forth tubes from the right end, the digits vary at the rate of 100,10 , and 1 times per second, respectively. In this case we will see a montage of ten digits from zero to nine in the first and second tubes, while in the forth tube we will be able to see every individual digit separately in the regular order. In the third tube an interesting phenomenon will be observed. We will not be able to read all ten digits in the regular order but we will miss some digits as if they do not

\footnotetext{
1 The author is very much indebted to Professor T. Oyama for his encouragement and many helpful comments about the paper in the whole course of preparing it. Thanks go also to S. Asakura and $\mathrm{R}$. Kawai for their helps in constructing the apparatus and collecting the data.
}

appear actually.

This phenomenon seems to reveal some intrinsic features of the visual mechanism of processing successive stimuli. At first, it demonstrates the existence of a limitation in the temporal differentiation of visual patterns which sequentially arrive at about the same portion of retina. Secondly, it suggests that there are some interactions among responses to sequential visual stimuli. In the present investigation, the nature of such interactions will mainly be studied, because the limitation in the above sense is considered to be greatly due to such interactions.

\section{General Method}

\section{Apparatus}

The apparatus used in the present study consists of the components shown in Fig. 1. The Decimal Counter (DCo) sends the rectangular wave pulses given by the Very Low Frequency Function Generator (FG) sequentially to the ten output channels, that is, the first pulse to the first output channel and the second to the second channel, and so on. The On-off Ratio Regulator controls the duration of the on-time in each cycle of the pulses before they are sent 
to DCio.

Each output channel of DCo is connected to each relay in the Relay Switching Circuit (RSC) and the combination between the output channels of DCo and the relays can be changed easily with the adjustment of the Connector. The Number Controller determines the number of pulses which are to be counted by DCo and it terminates the operation of DCo when the number of the signals counted by DCo reaches the predetermined number $(<10)$. RSG switches the circuits between the ten cathodes of the Nixie Tube (Okaya Musen CD-11/GR 201) and the power supply. Nixie Tube (NT) is a kind of glow tubes usually used as an indicator tube of the output of a digital circuit. It contains an anode and ten cathodes in shapes of digits. When the appropriate voltage is supplied between the anode and a cathode a red glow generates on the cathode and we can read clearly the corresponding digit in NT. We can present any digit in NT by selecting a cathode among the ten. The selection is made by the Connector between DCo and relays and also by the Scrambler, a rotary switch with multiple poles which changes connection between relays and cathodes of NT. This Scrambler has ten fixed patterns of connection which determine the order of presentation of digits.

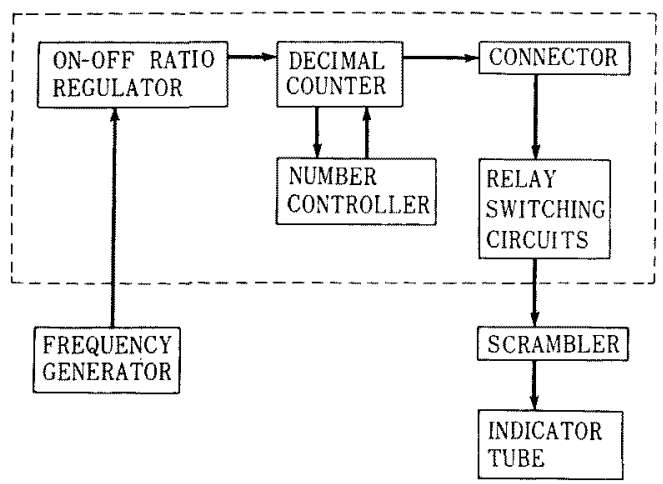

Fig. 1. A block diagram of the apparatus. The indicator tube was used to present 5 digits sequentially to the eye. It was attached to the stimulus presentation pannel of an observation box. Components enclosed by a dotted line were constructed with elements of Mitsubishi Logic Trainer, Type LT -3020 .
By the Connector and the Scrambler, the order of presentation of digits can be changed in numerous ways.

The components enclosed by a dotted line in Fig. 1 are constructed with elements of Mitsubishi Logic Trainer, Type LT-3020. The Logic Trainer is an apparatus consisting of a large number of electric elements for logic circuits, such as AND, OR, NOR and FLIPFLOP. All of these elements are transisterized, and each has its own input and output terminals. By connecting those elements according to various rules, we can form many kinds of logic circuits.

The NT (Nixie Tube) of our apparatus was attached to one of the stimulus presentation pannels of the observation box of a Dodge type two-channel tachistoscope. Two small neon lamps, served to indicate the fixation point (the center of the two), were located horizontally on another pannel and their light was reflected by a half mirror to reach the subject's eyes. The digits in the NT were seen between the image of the two neon lamps when they were lit. The neon lamps were always lit through the experimental session and the other portion of the observation box was completely dark. As ten cathodes in shapes of digits of NT were placed in different vertical planes, digits appeared in slightly different distances if they are observed binoculary. So the observation in the present study was done monoculary and a neutral filter whose density is 1.0 was placed in front of the eye to reduce the other irrelevant cues.

The luminance of the digits in NT was about $200 \mathrm{~mL}$ but the neutral filter in front of the eye reduced it to about $20 \mathrm{~mL}$. The difference in duration of the output from the input of relays of RSG in the apparatus ranged from -0.1 to $-2.6 \mathrm{msec}$ when they were operated by the rectangular wave pulses with duration of $10 \mathrm{msec}$ or more. The duration of the shortest pulse used as the input to the relays in the present study was $10 \mathrm{msec}$. Accordingly the duration of the outputs of relays and of the indicator tube controlled by them will range from 7.4 to 9.9 msec. The frequency of pulses generated by FG was calibrated with an oscilloscope and the on-time of each relay was also checked with it 
time to time. The visual angle of the height of each digit was about $2 \cdot 1^{\circ}$.

\section{General Procedure}

The whole apparatus was put in a dim experimental room. When the subject looked into the observation window, his head was covered by a black velvet hood to prevent the entrance of extraneous light to the eye. Instruction was given to the subject in the course of five minutes dark adaptation. At first, he was asked to fixate the middle of the two neon lamps. In Experiment I, only the whole report task was assigned. In Experiment II, the detection task was given in addition to the whole report task. In the whole report task, the subject was required to report all the digits he had recognized in a trial. The subject was not encouraged to guess, that is, to give five digits on each trial. He was encouraged not to report false digits. In the detection task, the subject was told a digit by the experimenter before a trial and was expected to answer whether the digit had been presented or not after the trial. In both experiments, the number of digits presented sequentially in a trial was always five. ${ }^{2}$ A trial was begun with a ready signal (click) and the first digit appeared three seconds after it. The rate or speed of the presentation of five digits were controlled by FG. Ten and five conditions of the presentation rate were used in $\mathbf{E x}$ periment I and Experiment II, respectively. Ten trials were run successively in each condition of the presentation rate in the whole report tasks. Every digit from zero to nine appeared once in every serial position in these ten trials in each condition of the presentation rate. The different five-digits series was used in each trial.

\footnotetext{
2 According to the results of the preliminary experiments, the stimulus series of five digits used in the present experiments were always smaller than short-term memory spans of the subjects when the series were presented in the rate of one digit per second.
}

\section{EXPERIMENT I}

\section{Experimental condition}

The conditions of the presentation rate were $50,33.3,25,16.6,12,10,8,6,5$ and 4 cps. The duration of one cycle, that is, cycle time $\tau(=1 / f)$, in each condition is $20,30,40,60$, $80,100,125,167,200$ or $250 \mathrm{msec}$. A digit is presented continuously during one cycle when the on-off ratio is $1: 0$, and it is presented in half period of one cycle when the ratio is $1: 1$. In the latter case, there exists a dark period of the same duration as the on-time of the digit between the presentation of one digit and the next. In both cases, the duration of the digit presentation becomes longer with the decrease of presentation rate. For example, under the 50 cps condition, a digit is presented for $20 \mathrm{msec}$ in the $1: 0$ on-off ratio condition and for $10 \mathrm{msec}$ in the 1:1 on-off ratio condition, while they are $200 \mathrm{msec}$ and $100 \mathrm{msec}$, respectively under $5 \mathrm{cps}$ condition.

The experiment was divided into two sessions according to the two conditions of on-off ratio. Each session took place in a different day for each subject. For half of the subjects one of the two conditions was used in the first session and for the other half the other condition was used first. The subject reported the whole digits he had been able to see in a series of digits presented in the indicator tube immediately after the end of each trial (the whole report task).

\section{Subject}

Four male and six female students majoring in psychology in Chiba University participated in this experiment. All had normal or corrected-to-normal vision and all had participated in the preliminary experiments of the present study. But they did not know any purpose of it.

\section{Results}

In Fig. 2 mean percentages of correct response are plotted with unfilled and halffilled dots as a function of $T(=1 / f)$. When the subjects reported a digit really 


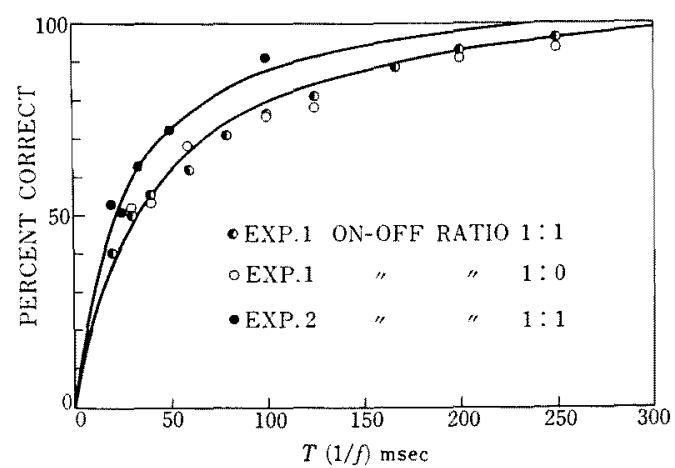

Fig. 2. Mean percentage of correct response as a function of cycle time $T$ (onset-to-onset interval between two successive digits). Unfilled and halffilled dots represent the results of the $1: 0$ condition and the $1: 1$ condition of on-off ratio of the whole report task in Exp. I, respectively. Filled dots represents the results of the detection task in Exp. II in which the on-off ratio was always $1: 1$.

\section{EXP.I ON-OFF RATIO $1: 0$}

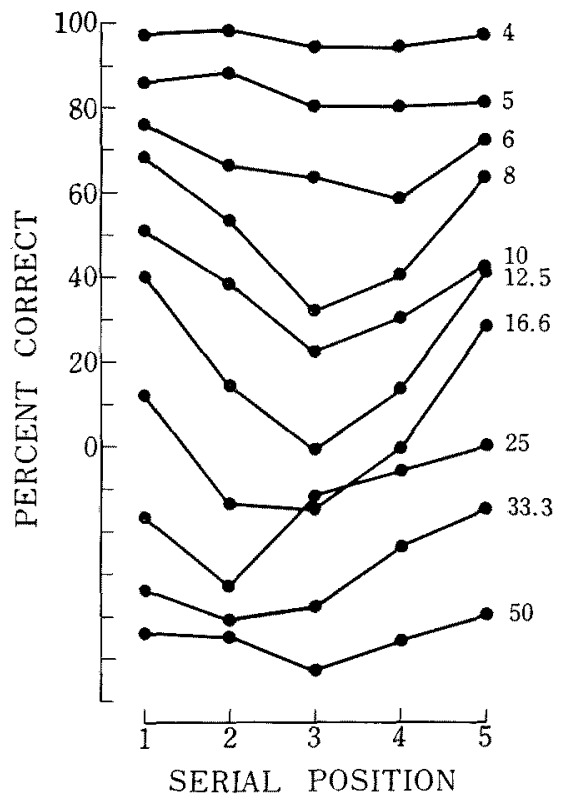

FIG. 3. The serial position effect in the whole report task under the $1: 1$ condition of on-off ratio in Exp. 1. Each point is mean percentage of correct response at each serial position for each condition of presentation rate, averaged over 10 trials and 10 subjects. All curves below the highest are shifted downward by 10 on the ordinate scale to avoid confusion. presented in a trial, the report was counted as a correct response without regard to the temporal order of the presentation of digits in the trial.

In Figs. 3 and 4, mean percentages of correct response are plotted as a function of the serial position. Each point in both figures represents the percentage of the correct responses to 100 possible correct responses in each serial position. The parameters in both figures are the ten values of presentation rate in cps. It must be noticed that all the curves below the highest in both figures are shifted successively downward by 10 on the ordinate scale to avoid confusion. Almost all curves show differences caused by the serial positions and remarkable tendency that the

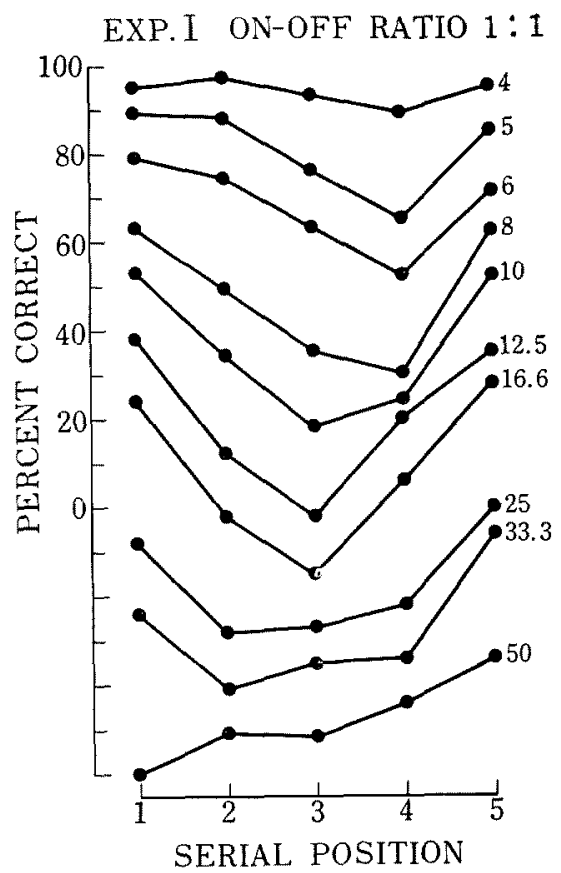

Frg. 4. The serial position effect in the whole report task under the $1: 0$ condition of on-off ratio in Exp. I. Each point is mean percentage of correct response at each serial position for each condition of presentation rate, averaged over 10 trials and 10 subjects. All the curves below the highest are shifted downward by 10 on the ordinate scale to avoid confusion. 
shape of the curve changes according to the change in the presentation rate. The minimum value of the percentage of the correct responses in each curve gradually shifts from the second to the fourth in serial position as the presentation rate decreases from 33.3 to $4 \mathrm{cps}$, except the $50 \mathrm{cps}$ condition in either on-off ratio condition. For the analysis of variance inverse sine transformation was applied to the mean percentage of correct response of each subject over 10 trials under each condition. Factors used in the analysis were on-off ratio (2 levels), serial position (5 levels), and presentation rate (10 levels.) The effect of on-off ratio was not significant. The effects of serial position and presentation rate were highly significant $(F=56.44, d f=$ 4/900, $P<0.01: F=72.38, d f=9 / 900, P<$ 0.01 : respectively). As to the interaction terms, serial position $\times$ presentation rate and on-off ratio $\times$ presentation were significant $(F=3.30, d f=36 / 900, P<0.01: F=$ 5.68, $d f=9 / 900, P<0.01$ : respectively). To test the effect of the serial position the Friedman two-way analysis of variance (5 serial position, 10 subjects) was applied to the data of individual subjects on percentage of correct response in five serial positions, for 10 conditions of presentation rate. Under all conditions of presentation rate the serial position effect is significant beyond the $5 \%$ level at least except under $4 \mathrm{cps}$ condition in both conditions of on-off ratio and $5 \mathrm{cps}$ condition in the on-off ratio of $1: 1$. The general tendency is the same in both on-off ratio conditions. The slight differences shown particulary in low frequencies are not clear enough to tell any systematic differences between the two conditions of on-off ratio.

The digits in the indicator tube used in this experiment do not seem to be homogenious in the easiness of recognition. In this experiment each digit appeared five times in each condition of presentation rate. Correct responses for each digit were summed over ten subjects and ten conditions of presentation rate. Percentages of correct responses for the 500 possible correct responses were: $75.8,78.0,69.0,60.0$, $80.2,58.2,65.6,87.0,74.8$, and $52.0 \%$ for digit $0,1,2,3,4,5,6,7,8$, and 9 , respectively. The percentages ranged from $52.0 \%$ for digit 9 to $87.0 \%$ for digit 7 , and the mean for ten digits was $70.1 \%$. From the results, it can hardly be said that each digit is homogenious in its easiness of recognition. But the experimental design was made to counterbalance the effect due to the differences in easiness of recognition by presenting every digit equal number of times in every serial position in every condition of presentation rate and of on-off ratio. Then, we can disregard the differences found in the percentage of correct responses for individual digits in discussing the results of the present experiment.

\section{EXPERIMENT II}

The well-known serial position effect on verbal rote learning can be considered as the effect occurred in memory process. But the effect found in Experiment I seems to be an effect the origin of which is in visual process. In Experiment II, an effort was made to lighten the memory load of the subject and to extract the effect in the visual process as purely as possible. For this purpose the detection task which is a vertion of the partial report task used by Averbach and Sperling (1961) was assigned to each subject. In the task, the subject was told a digit before each trial by the experimenter and asked to detect the digit (target) in the trial, where 5 digits were sequentially presented as in Experiment I. Immediately after the end of a trial, the subject reported whether he detected the target or not. After the detection task experiment, the whole report task was also conducted using the same subject and the same conditions of presentation rate to ascertain whether the effects found in Experiment I were found again in the subjects and conditions of Experiment II. 


\section{Experimental condition}

The presentation rates in Experiment II were $10,20,30,40$ and $50 \mathrm{cps}$. The on-off ratio was always 1:1. The number of digits presented in one trial was constantly five, but digits used in each trial were systematically varied to counterbalance the frequencies of presentation of ten digits in each condition of the experiment.

In the detection task, 100 trials were run for each subject under each condition of presentation rate. Totally, 500 trials were run for each subject in this task II. Each of ten digits was used ten times as the trarget in 100 trials under each conditions of presentation. And each of ten digits was used once as the target in each of 5 serial positions. Consequently, in 50 of the 100 trials, a digit which was not contained in the 5 digit series of each trial was chosen as the target, that is, those 50 trials were so called blank experiments. These 50 blank experiments were dispersed randomly in the 100 trials. Five subjects and five conditions of presentation rate were combined following a Latin square chosen randomly.

In the whole report task, ten trials were run for each subject under each presentation rate condition and the subjects were instructed to report all the digits he recognized in each trial. All other conditions were the same as those of the detection task. Of course, a different Latin square from that in the detection task was used to arrange five subjects and five conditions in the whole report task.

\section{Subject}

Four male and one female students majoring in psychology in Chiba University participated in both tasks. They had not participated in Experiment I. All had normal or correctedto-normal vision.

\section{Results}

Mean percentage of correct response in the detection task is plotted by filled dots in Fig. 2 as a function of $T(=1 / f)$. As in Experiment I mean percentage of correct response is a negatively accerated increasing function of $T$. But the curve grows faster than that of the curves in Experiment I. The right answers in the blank experiments (correct rejection) were not included in the correct responses because they did not show any systematic variation according to the presentation rate and only a small number of errors were found in the blank experiment.

In Fig. 5, the mean percentage of correct response in the detection task is plotted as a function of the serial position. The parameters in the figure are values of presentation rate in cps. Each point in the figure represents the percentage of the correct responses to 50 possible correct responses ( 5 subjects $\times 10$ trials) in each serial position. All the curves below the highest are shifted successively downward by 10 on the ordinate scale to avoid confusion. The general tendency in the curves appears to be the same as the results in Experiment $I$ shown in Fig. 3 and 4. The shift of the minimum value of the percentage of correct responses in each

\section{EXP.II ON-OFF RATIO $1: 1$}

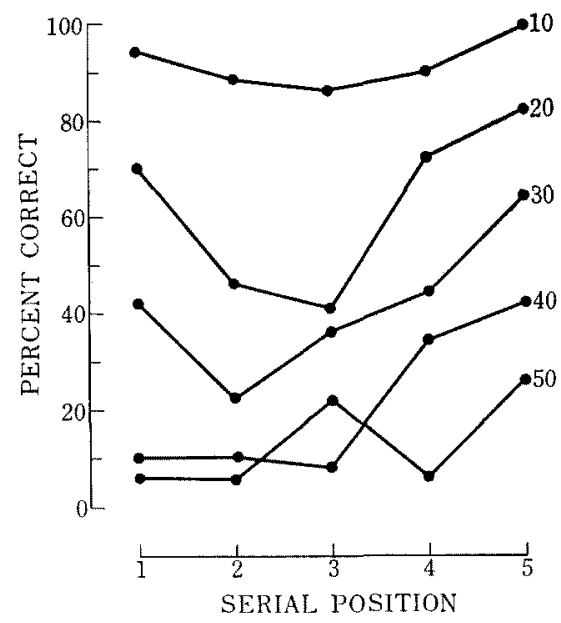

Fig. 5. The serial position effect in the detection task under the 1: 1 condition of on-off ratio in Exp. II. Each point is mean percentage of correct response at each serial position for each condition of presentation rate, averaged over 10 trials and five subjects. All the curves below the highest are shifted by 10 on the ordinate scale to avoid confusion. 
curve with the decrease of the presentation rate is found in this figure again. For the analysis of variance inverse sine transformation was applied to the mean percentage of correct response of each subject over 10 trials under each condition. Factors in the analysis were serial position (5 levels) and presentation rate (5 levels). The effects of serial position and presentation rate were both highly significant $\quad(F=10.81, \quad d f=4 / 100, \quad P<0.01$ : $F=29.62, \quad d f=4 / 100, \quad P<0.01$ : respectively). Serial position $\times$ presentation rate was not significant. According to the Friedman two-way analysis of variance, the serial position effect was significant for 20 and $30 \mathrm{cps}$ conditions beyond $1 \%$ level.

The results of the whole report task was very similar to those of Experiment $I$ as to the relation in Fig. 2 and the tendency in the serial position effect was also very similar to those of Experiment I and of detection task in Experiment II. For this reason, the results of the whole report task in Experiment II are not shown by a figure in this paper. The serial position effect was significant for 20,30 and $40 \mathrm{cps}$ conditions beyond $1 \%$ level according to the Friedman two-way analysis of variance.

\section{Discussion}

\section{Serial position effect}

The most remarkable findings in the present experiments are the facts that the percentage of correct response differs among serial positions and that the serial position for the minimum percent correct value shifts gradually from the second to the forth position with the decrease of the presentation rate from $33.3 \mathrm{cps}$ to $4 \mathrm{cps}$. And it is also impressive that those effects were found in the detection task (Exp. II), too. This fact suggests that the origin of those effects exists in visual process, not in memory process.

As to the sources of the serial position effect, it will be most natural to suppose that two kinds of inhibitions are acting upon responses to individual members in the stimulus series. In the field of the verbal rote learning the serial position effect is often explained by the two kinds of inhibitions, that is, proactive inhibition and retroactive inhibition. A good example of such theories is seen in Ono (1958). A similar model may be applicable to the serial position effect in the processing of the sequential visual stimuli shown in this study, though it occurs in much shorter time than the serial position effect in verbal learning. In the present case, those two inhibitions are supposed to be forward masking effect and backward masking effect.

From the evidences about so called visual masking by pattern (Schiller, 1966 ; Kahneman, 1968), it can be assumed that each response in a level of visual process to each stimulus always give forward and backward masking effects to preceding and following responses, even if the effects can not be found experimentally when the intensity of the stimulus is very weak. Let us assume also that visual masking effects are summated. These mean that a response produced by a visual stimulus is affected by the total masking effect which is the sum of the masking effects of several preceding and following stimuli when those stimuli are given in rapid succession.

From those assumptions, we can deduce some predictions. First, the response in the first serial position is affected only by backward masking and that in the last serial position, only by forward masking, while the responses in other serial positions are affected by both types of masking. Secondly, a curve which represents the functional relationship between mean percent correct value and the serial position will reflect the total visual masking effect by which each response in each serial position is affected. Lastly and dependently on above two predictions, the characteristics of the time courses of visual forward and backward masking will be 
estimated from the shape of the serial position curve.

Shown in the Fig. 3 are the serial position curves for $1: 1$ condition of onoff ratio in Experiment I. In the figure the shift of the minima among serial position curves is seen most clearly. Following two points are to be noticed. 1) The minimum point is located at the first serial position in the $50 \mathrm{cps}$ condition of presentation rate and then shifts to the second position in the $33.3 \mathrm{cps}$ and $25 \mathrm{cps}$ conditions. In the $16.6,12.5$, and $10 \mathrm{cps}$ conditions it is in the third position, the middle one. Finally it reaches to the fourth position in the $8,6,5$, and $4 \mathrm{cps}$ conditions. (About the same tendency is seen in Figs. 4, and 5 except some high and low conditions of the presentation rate). 2) Mean percent correct value in the first position is lower than that of the fifth (end) position in the $16.6 \mathrm{cps}$ and higher conditions. In the lower conditions of presentation rate that value is almost same for the first and end positions or sometimes inverse relations appear. According to the discussion made in the above paragraph, it can be concluded that with relatively high rates of presentation, the total amount of the backward masking effect by following responses upon the response to the first stimulus is greater than that of the forward masking effect by preceding responses upon the response to the last stimulus. It seems that backward masking has greater inhibitory effect than forward masking within the time interval from onset of the stimulus up to $1 / 16.6 \mathrm{sec}$. In other words, backward masking function has greater value than forward masking function at the beginning part of their time course. And if the rate of decay is the same in both masking functions, the first serial position will take always smaller mean percent correct value than the last serial position and the shift of minima in serial position curves will not occur. Consequently, the facts 1) and 2) indicate that the rate of decay of backward masking must be faster than that of forward masking, and that the forward masking must survive longer than backward masking. For explanatory purpose, we may assume exponential function, $I=a \exp (-b t)$, as the masking function, where $I$ is the magnitude of masking and $t$ is the time interval between the onset of two consecutive stimuli, $a$ and $b$ are constants and $a$ determines the value when $t=0$ and $b$, the rate of decay. Then we can summarize above discussion as follows: both constants $a$ and $b$ are greater for backward masking than for forward masking. If $a$ and $b$ are determined adequately in this type of exponential function and the total amount of masking which each response in each serial position will receive is calculated, the same type of changes in serial position curves as those found in the present study can be demonstrated when the ordinate is expressed by the inverse of the total amount of masking.

There are some evidences which support the proposition we made about the masking function. It is shown in some previous investigations that the forward masking is generally more effective than backward masking (Kinsbourne \& Warrington, 1962a, 1962b; Schiller, 1966; Eriksen, 1966). The predicted slower decay of forward masking is supported by the results of these investigations, though we have not found any evidences to support the prediction that backward masking grows faster than forward masking.

Very recently, some investigators reported serial position effects similar to that found in the present study, and tried to explain these phenomena. Haber and Nathanson (1969) attributed the serial position effect they found to visual masking, although their analysis and description were not enough to compare the result between their study and the present study.

Katz, Schoenberg and Mayzner (1970) and Mayzner, Tresselt, Tabenkin, Didner and Helfer (1969), Mayzner, Tresselt, Checksana and Hoenig (1970) found the 
same kind of serial position effect as that of the present study for sequentially presented digits. Especially in the curves presented in the latter, we can find not only the serial position effect but also the shift of minima from the second to the third serial position for five-digits string when the stimulus onset-to-onset interval among the stimuli was changed from $20 \mathrm{msec}$ to $100 \mathrm{msec}$ in $20 \mathrm{msec}$ step, although they did not give enough discussion on these facts. Their 20 msec onset-to-onset interval corresponds to the $50 \mathrm{cps}$ condition of presentation rate in the present study and their $100 \mathrm{msec}$, to the $10 \mathrm{cps}$ condition, except the fact that in their studies the stimulus duration was always $10 \mathrm{msec}$. In some experiments in the series of investigation made by Mayzner et al., (Mayzner et al., 1969; Katz et al., 1970; Mayzner et al., 1970) especially of Katz, et al. (1970) the shapes of serial position curves are somewhat different from those of the present study. The main discrepancy is in mean percentage of correct response values for the first serial position. In their data the first position often shows the highest value of that measure for any onset-to-onset interval. This tendency is more eminent in series with longer digits string (they used $2,3,4,5,6$ or 7 digits strings). This difference of results between their study and the present study may be mainly due to the difference of the task assigned to subjects in the two studies. Their subjects had to report the correct digits in their correct locations, while the subjects in the present study did not have to report locations of digits.

In their studies, no attempt was made to separate visual process from memory process by some experimental operations, while it was done in the present experiments using the whole report task and the detection task. The experimental operations in which the whole report task is used in one experiment and the detection task in the other as in the present study, can be regarded as a pair of converging opera- tions to eliminate the effects in the memory process and to extract the effects in the visual process as purely as possible. Those operations are important to clarify the relationship between the serial position effect in processing sequential visual stimuli and visual masking.

Some uncontrolled variables due to immediate memory process seem to affect their results. But on the whole, their results support the above discussions of the present study.

\section{On-off ratio}

In the analysis of variance applied to data of Experiment I, the effect of on-off ratio was not significant. And on-off ratio $\times$ serial position was also insignificant. This means that the dark period between the stimuli is not an important factor but the onset-to-onset interval between the stimuli is one of principal variables in processing sequential stimuli. It is an interesting coincidence that the duration of short-term visual storage for abovethreshold stimuli is almost independent of the stimulus duration (Haber \& Nathanson, 1969; Haber \& Standing, 1969). However on-off ratio $\times$ presentation rate was significant. From Fig. 2 it is found that for shorter cycle time than $80 \mathrm{msec}$, mean percent correct values for the $1: 0$ on-off ratio condition tended to be somewhat greater than those for the $1: 1$ condition, and for faster cycle time than 100 msec the former values are smaller than the latter values. For this reason, some effects of the duration of stimuli can not be denied, but they might not be so large if they exist.

\section{Differences between the whole report task and the detection task}

As shown in Fig. 2, percent correct values for the detection task are always greater than those of the whole report task when they are compared for the same or nearly the same cycle time. The difference between the results of the two tasks 
is considered to indicate the role of the short-term memory process in the situation. In the present study the limitation caused by the short-term memory span does not matter because the stimulus series is within the span (see footnote 2). The problem is the time to be needed to store each sequential stimuli in the short-term memory for verbal reporting. In the whole report task, digits themselves must be memorized in the short-term memory but in the detection task, results of some binary decisions are only material to be memorized. The load on the short-term memory is lighter in the latter than in the former. Many subjects sometimes said in the whole report task, "I am sure I saw some more digits, but I can not say what they were." The processing time per digit to make a correct verbal report must be longer for the whole report task than for the detection task.

\section{REFERENGES}

Averbach, E., \& Sperling, G. 1961 Short-term storage of information in vision. In $\mathrm{C}$. Cherry (Ed.), Information theory. London: Butterworth.

Eriksen, C. W. 1966 Temporal luminance summation effects in backward and forward masking. Perception \& Psychophysics, 1, 87-92.

Haber, R. H., \& Nathanson, L. D. 1969 Processing of sequentially presented letters. Perception \& Psychophysics, 5, 359-361.

Haber, R. H., \& Standing, L. G. 1969 Direct measures of short-term visual storage. Quart.
J. exp. Psychol., 21, 43-54.

Kanneman, D. 1968 Method, findings, and theory in studies of visual masking. Psychol. Bull., 70, 404-425.

Katz, M., Schoenberg, K. M., \& Mayzner, M. S. 1970 Visual information processing of sequentially presented inputs: II. Effects of list length and interstimulus interval values on subspan storage and retrieval mechanisms. Perception \& Psychophysics, 7, 149-152.

Kinsbourne, M., \& Warrington, E. K. 1962 a The effect of an after-coming random pattern on the perception of brief visual stimuli. Quart. J. exp. Psychol., 14, 223-234.

Kinsbourne, M., \& Warrington, E. K. $1962 \mathrm{~b}$ Further studies on the masking of brief visual stimuli by a random pattern. Quart. J. exp. Psychol., 14, 235-245.

Mayzner, M. S., Tresselt, M. E., Tabenkin, N., Didner, R., \& Helfer, M. S. 1969 Visual information processing of sequentially presented inputs: I. Effects of input timing on sub-span storage and retrieval mechanisms. Perception E Psychophysics, 5, 297-302.

Mayzner, M. S., Tresselt, M. E., Gheckes, J., \& Hoenig, H. A. 1970 Visual information processing of sequentially presented inputs : III. Further effects of list length and interstimulus interval values on sub-span storage and retrieval mechanisms. Perception \& Psychophysics, 7, 294-296.

ONo, S. 1958 A mathematical analysis of serial verbal learning. Jap. psychol. Res., 1, 1-26.

Schiller, P. H. 1966 Forward and backward masking as a function of relative overlap and intensity of test and masking stimuli. Perception $E^{\circ}$ Psychophysics, 1, 161-164.

(Received Oct. 17, 1970) 